\title{
History of lower-limb complications and risk of cancer death in people with type 2 diabetes
}

\author{
Kamel Mohammedi ${ }^{1,2,3^{*}} \mathbb{D}$, Stephen Harrap ${ }^{4}$, Giuseppe Mancia ${ }^{5}$, Michel Marre ${ }^{6,7,8}$, Neil Poulter ${ }^{9}$, \\ John Chalmers ${ }^{10,11}$ and Mark Woodward ${ }^{10,11,12,13}$
}

\begin{abstract}
Background: Individuals with diabetes and lower-limb complications are at high risk for cardiovascular and all-cause mortality, but uncertainties remain in terms of cancer-related death in this population. We investigated this relationship in a large cohort of people with type 2 diabetes.

Methods: We used data from the Action in Diabetes and Vascular Disease: PreterAx and DiamicroN Modified-Release Controlled Evaluation (ADVANCE) study. The primary outcome was adjudicated cancer death; secondary outcomes were overall and site-specific incident cancers, determined according to the International Classification of Diseases Code (ICD-10). We compared outcomes in individuals with (versus without) a baseline history of lower-limb complications (peripheral artery disease (PAD) or sensory peripheral neuropathy) using Cox regression models.
\end{abstract}

Results: Among 11,140 participants (women 42\%, mean age 66 years), lower-limb complications were reported at baseline in 4293 (38\%) individuals: 2439 (22\%) with PAD and 2973 (27\%) with peripheral neuropathy. Cancer death occurred in 316 (2.8\%) participants during a median of 5.0 (25th-75th percentile, 4.7-5.1) years of follow-up corresponding to 53,550 person-years and an incidence rate of 5.9 (95\% Cl 5.3-6.6) per 1000 person-years. The risk of cancer death was higher in individuals with (versus without) lower-limb complication [hazard ratio 1.53 ( $95 \% \mathrm{Cl}$, $1.21-1.94), p=0.0004]$, PAD [1.32 (1.02-1.70), $p=0.03$ ] or neuropathy (1.41 (1.11-1.79), $p=0.004]$, adjusting for potential confounders and study allocations. PAD, but not neuropathy, was associated with excess risk of incident cancers.

Conclusions: PAD and peripheral neuropathy were independently associated with increased 5 -year risk of cancer death in individuals with type 2 diabetes. PAD was also associated with increased risk of incident cancers. Our findings provide new evidence on the non-cardiovascular prognostic burden of lower-limb complications in people with type 2 diabetes.

Keywords: Cancers, Cancer death, Lower-limb complication, Peripheral arterial disease, Peripheral neuropathy, Type 2 diabetes

\section{Background}

Peripheral artery disease (PAD) and peripheral sensory neuropathy are major lower-limb complications frequently observed in patients with diabetes [1-5]. These are the leading causes of non-traumatic lower-extremity

*Correspondence: km.mmohammedi@gmail.com

${ }^{1}$ Department of Endocrinology, Diabetes and Nutrition, Bordeaux University Hospital, Bordeaux, France

Full list of author information is available at the end of the article amputation, at least 8 times more common in people with diabetes than in persons without diabetes $[6,7]$. Lower-limb complications are associated with major disability, worsening quality of life and huge impacts on health care systems and societies $[1,8,9]$. They are also associated with a dramatic reduction in life expectancy, despite major improvements in medical care during recent decades [3, 10-13]. The excess risk of premature death observed in patients with diabetes and lowerlimb complications may not be completely attributable 
to cardiovascular disease, as other non-cardiovascular conditions may also be involved [14].

Cancer is a major public health problem worldwide, and is the second leading cause of death [15]. Growing data suggest an excess risk for cancer death among adults with diabetes, compared with others [16-19]. The risk of cancer increased in people with cardiovascular disease as did the risk of cardiovascular disease in cancer patients [20]. Only few studies have evaluated the risk for cancer death according to vascular complications in people with diabetes, and have reported inconsistent findings [21-23]. As far as we are aware, the relationship between lower-limb complications and cancer death has not been investigated in patients with diabetes. In the present study, we aimed to evaluate the incidence and the relative risk of cancer death according to the baseline history of lower-limb complications among patients with type 2 diabetes in the Action in Diabetes and Vascular Disease: PreterAx and DiamicroN Modified-Release Controlled Evaluation (ADVANCE) study.

\section{Methods \\ Participants}

ADVANCE was a large multicentre international randomized trial conducted in patients with type 2 diabetes to test the effects of intensive glucose control using a gliclazide-MR based regimen and blood pressure treatment using a fixed-dose combination of perindopril and indapamide on the incidence of major microvascular and macrovacular events [24]. The design and clinical characteristics of participants in ADVANCE have been published previously [24-26]. Briefly, patients with type 2 diabetes mellitus, and at least one additional risk factor for cardiovascular disease, were randomly assigned in a 2 X 2 factorial design to: (i) gliclazide (modified release)-based intensive glucose-control regimen, targeting an $\mathrm{HbA} 1 \mathrm{c}$ of $\leq 6.5 \%$, or to standard glucose control, with targets and regimens based on local guidelines, and (ii) a fixed-dose combination of perindopril $(4 \mathrm{mg})$ and indapamide $(1.25 \mathrm{mg})$ or matching placebo. Participants were enrolled between 2001 and 2003; they were seen 3, 4, and 6 months after randomisation, and subsequently, every 6 months until June 2007 (for blood-pressure-lowering comparison) [25]. The follow-up in the randomized glucose-control regimen was continued for an additional 6 months, until January 2008 with a different follow-up schedule as published previously $[25,26]$. The protocol of the ADVANCE trial was approved by the Institutional Ethics Committee of each participating centre and all participants provided written informed consent.

\section{Definition of lower-limb complications at baseline}

A history of lower-limb complication was defined as the presence at baseline of PAD or peripheral neuropathy. PAD was defined as the presence of at least one of following conditions: lack of peripheral pulse (dorsalis pedis or posterior tibial) palpation, requirement of lower-limb revascularisation (surgery, angioplasty or emergency thrombolysis), or lower-extremity amputation of at least one digit, thought to be due to arterial insufficiency. Peripheral sensory neuropathy was defined as the presence at baseline of at least two neurological abnormalities [27]: disturbance of the light touch sensation, abolition of ankle or knee reflex or chronic (at least 6 weeks) foot ulceration. Data were collected by physicians for each participant based on interview and clinical examination, and reported in the case report forms.

\section{Definition of other conditions at baseline}

History of coronary artery disease (CAD) was defined as the presence at baseline of at least myocardial infarction, coronary artery bypass graft, percutaneous transluminal coronary angioplasty, or hospital admission for unstable angina. History of cerebrovascular disease was defined as the presence at baseline of stroke or transient ischaemic attack. Diabetic retinopathy was defined as the presence of at least one of the following conditions: proliferative retinopathy, macular oedema, requirement of retinal laser photocoagulation therapy, or diabetes-related blindness. History of diabetic kidney disease (DKD) was defined as urinary albumin-to-creatinine ratio $(\mathrm{ACR})>30 \mu \mathrm{g} / \mathrm{mg}$ or estimated glomerular filtration rate (eGFR, computed using the Chronic Kidney Disease Epidemiology Collaboration equation) $<60 \mathrm{ml} / \mathrm{min} / 1.73 \mathrm{~m}^{2}$.

Region of origin was categorized as 3 groups: Asia (Philippines, China, Malaysia, and India), established market economies (Australia, Canada, France, Germany, Ireland, Italy, Netherlands, New Zealand, and United Kingdom) and Eastern Europe (the Czech Republic, Estonia, Hungary, Lithuania, Poland, Russia, and Slovakia). Cognitive function was estimated by the mini-mental state examination (MMSE) score and considered as normal (MMSE score $\geq 28$ ) or reduced (MMSE score $<28$ ). Education accomplishment was defined as age at completion of the highest level of formal education, and categorized as basic ( $\geq 16$ years) or low ( $\leq 15$ years).

\section{Primary and secondary endpoints}

The primary endpoint for this analysis was cancer death occurring during follow-up. The cause of each death, including cancer-related death, was adjudicated by an independent endpoint committee, blinded to study allocations. The adjudication committee reviewed source 
documentations for all individuals who died during follow-up. The secondary endpoints were overall and site-specific incident cancers, defined as the first cancer diagnosed after entry to the study according to the International Classification of Diseases Code Tenth Revision (ICD-10). Diagnostic codes used in the study are presented in Additional file 1: Table S1. Incident cancers were collected systematically for all participants during the scheduled study visits from case report forms, and from reports of serious adverse events, without adjudication. Information about the occurrence of all serious adverse events was reported at the time of occurrence between visits. When serious adverse events occurred, the responsible investigator of each centre ensured that the event was reported immediately by completing a Serious Adverse Events Form. The Data and Safety Monitoring Committee regularly reviewed all such events for each centre. The effect of intensive (versus conventional) glucose control on cancer death in ADVANCE has been previously reported [28].

\section{Statistical analyses}

Quantitative variables were expressed as mean (SD), or median (25th, 75th percentiles) for those with skewed distributions. Categorical parameters were expressed as numbers and percentages.

Cox proportional hazards regression models were used to estimate hazard ratios (HR) and their 95\% confidence intervals (CI) for risk of outcomes according to our three index exposures. We compared the incidence of cancer death according to study allocations: perindopril/indapamide combination (versus placebo) or both intensive glucose control and active blood pressure treatment (versus standard glucose control and placebo group).

Kaplan-Meier curves were used to plot the cumulative incidence of cancer death during follow-up according to the baseline history of lower-limb complication, PAD or peripheral neuropathy at baseline. Survival curves were compared using the log-rank test. Cox proportional hazards regression models were fitted to estimate the risk of outcomes during follow-up according to the history of lower-limb complication at baseline. Analyses were adjusted for baseline age, sex, region of origin and study allocations (model 1), and for model 1 plus any potential confounders: duration of diabetes, body mass index, waist circumference, systolic and diastolic blood pressure, HbA1c, urinary ACR, eGFR, total cholesterol, HDL cholesterol, triglycerides, MMSE score, education accomplishment, history of ever or current smoking, history of past or current alcohol drinking, history of CAD, cerebrovascular disease, diabetic retinopathy or dementia, and use of metformin, insulin, antihypertensive, statins or antiplatelet therapies (model 2). Continuous variables supposed to have U-shaped relationships with death (age, body mass index, waist circumference, systolic and diastolic blood pressure, HbA1c, and eGFR) were introduced within the model with their squared values.

We tested for interaction between PAD and peripheral neuropathy in their association with cancer death. The proportional hazards assumption was checked using the Schoenfeld residuals method.

We performed a series of sensitivity analyses to evaluate the risks of endpoints according to: 1 / the history of lower-limb complications at baseline using an alternative definition of lower-limb complications: the presence of at least one of the following conditions: PAD (lack of peripheral pulse palpation or requirement of lower-limb revascularisation), peripheral neuropathy (at least two neurological abnormalities: disturbance of the light touch sensation, abolition of ankle or knee reflex), or tissue or limb loss (foot ulceration or lower-extremity amputation); $2 /$ peripheral neuropathy defined alternatively as the presence of at least one neurological abnormality (disturbance of the light touch sensation, abolition of ankle reflex or abolition of knee reflex) or a history of foot ulceration. We evaluated the associations between lower-limb complications and the risk of cancer death in people who have smoked cigarettes regularly (most days for at least a year) and those who have never smoked. Also, we tested the association between lower-limb complications and the risk of cardiovascular and all-cause death. Finally, we used the Fine and Gray method to estimate the subdistribution Hazard ratio for cancer death, while accounting for the competing risk of death from non-cancer causes further to adjusting as in model 2 [29].

Statistics were performed using SAS software, version 9.3 (SAS Institute, Cary, NC, USA, www.sas.com) and Stata software version 15.1 (StataCorp, TX, USA, http:// www.stata.com). Two-sided $P$ values $<0.05$ were considered statistically significant.

\section{Results \\ Clinical characteristics at baseline}

Among 11,140 ADVANCE participants, 4733 (42\%) were women, 4136 (37\%) from Asia, 4862 (44\%) from established market economies and 2142 (19\%) from eastern Europe. Their mean (SD) age and duration of diabetes were $66(6)$ and $7.9(6.3)$ years, respectively, and the mean (SD) HbA1c was $7.5(1.6 \%)$ at baseline (Table 1). A history of lower-limb complication was reported at baseline in 4293 (38\%) participants: 2439 (22\%) with PAD and 2973 (27\%) with peripheral neuropathy. PAD and peripheral neuropathy were present simultaneously in 1119 $(10 \%)$ individuals. Characteristics of participants according to a history of PAD or peripheral neuropathy at baseline are displayed in Table 1. 
Table 1 Characteristics of participants according to history of peripheral artery disease or peripheral neuropathy at baseline

\begin{tabular}{|c|c|c|c|c|c|}
\hline & \multirow[t]{2}{*}{ Overall } & \multicolumn{2}{|c|}{ Peripheral artery disease } & \multicolumn{2}{|c|}{ Peripheral neuropathy } \\
\hline & & No & Yes & No & Yes \\
\hline Number & 11,140 & 8701 & $2439(22)$ & 8167 & $2973(27)$ \\
\hline \multicolumn{6}{|l|}{ Gender, n (\%) } \\
\hline Women & $4733(42)$ & 3816 & $917(19)$ & 3580 & $1153(24)$ \\
\hline Men & $6407(58)$ & 4885 & $1522(24)$ & 4587 & $1820(28)$ \\
\hline \multicolumn{6}{|l|}{ Region of origin, $\mathrm{n}(\%)$} \\
\hline Asia & $4136(37)$ & 3755 & $381(9)$ & 3426 & $710(17)$ \\
\hline Established market economies & $4862(44)$ & 3225 & $1637(34)$ & 3260 & $1602(33)$ \\
\hline Eastern Europe & $2142(19)$ & 1721 & $421(20)$ & 1481 & $661(31)$ \\
\hline Age, years: mean (SD) & $66(6)$ & $65(6)$ & $67(7)$ & $65[6]$ & $67(7)$ \\
\hline Duration of diabetes, years: mean (SD) & $7.9(6.3)$ & $7.8(6.2)$ & $8.4(6.8)$ & $7.6(6.2)$ & $8.7(6.6)$ \\
\hline Body mass index, kg/m²: mean (SD) & $28(5)$ & $28(5)$ & $29(5)$ & $28(5)$ & $29(6)$ \\
\hline Waist circumference, cm: mean (SD) & $98(13)$ & $98(13)$ & $102(13)$ & $97(13)$ & $102(13)$ \\
\hline Systolic blood pressure, mmHg: mean (SD) & $145(21)$ & $145(21)$ & $146(21)$ & $145(21)$ & $146(21)$ \\
\hline Diastolic blood pressure, mmHg: mean (SD) & $81(11)$ & $81(11)$ & $80(10)$ & $81(11)$ & $80(11)$ \\
\hline \multicolumn{6}{|l|}{ Mini-mental state examination } \\
\hline Overall MMSE score, mean (SD) & $28(2)$ & $29(2)$ & $28(2)$ & $29(2)$ & $28(2)$ \\
\hline Participants with normal MMSE score, n (\%) & $8689(78)$ & 6900 & $1789(21)$ & 6480 & $2209(25)$ \\
\hline Participants with reduced MMSE score, n (\%) & $2451(22)$ & 1801 & $650(27)$ & 1687 & $764(31)$ \\
\hline \multicolumn{6}{|l|}{ Education accomplishment } \\
\hline Age at completion, years: mean (SD) & $18(7)$ & $19(7)$ & $18(7)$ & $19(7)$ & $18(7)$ \\
\hline Participants with basic education, n (\%) & $7116(64)$ & 5809 & $1307(18)$ & 5402 & $1714(24)$ \\
\hline Participants with low education, n (\%) & $4024(36)$ & 2892 & $1132(28)$ & 2765 & $1259(31)$ \\
\hline \multicolumn{6}{|l|}{ HbA1c, mean (SD) } \\
\hline (\%) & $7.5(1.6)$ & $7.5(1.6)$ & $7.4(1.4)$ & $7.5(1.6)$ & $7.5(1.5)$ \\
\hline $\mathrm{mmol} / \mathrm{mol}$ & $59(17)$ & $59(17)$ & $58(15)$ & $59(17)$ & $59(16)$ \\
\hline Urinary ACR, $\mu \mathrm{g} / \mathrm{mg}$ : median $\left(25^{\text {th }}, 75^{\text {th }}\right.$ percentiles $)$ & $1(1,2)$ & $1.0(1.0,2.0)$ & $1.0(1.0,2.0)$ & $1.0(1.0,2.0)$ & $1.0(1.0,2.0)$ \\
\hline eGFR, ml/min/1.73m²: mean (SD) & $74(17)$ & $75(17)$ & $71(18)$ & $75(18)$ & $72(17)$ \\
\hline Serum total cholesterol, mmol/l: mean (SD) & $5.2(1.2)$ & $5.2(1.2)$ & $5.0(1.1)$ & $5.2(1.2)$ & $5.1(1.2)$ \\
\hline Serum HDL cholesterol, mmol/l: mean (SD) & $1.2(0.3)$ & $1.3(0.3)$ & $1.2(0.3)$ & $1.3(0.4)$ & $1.2(0.3)$ \\
\hline Serum LDL cholesterol, mmol/l: mean (SD) & $3.1(1.0)$ & $3.1(1.0)$ & $3.0(1.0)$ & $3.1(1.0)$ & $3.0(1.0)$ \\
\hline Serum triglycerides, mmol/l: median (25th, 75 th percentiles) & $1.6(1.2,2.3)$ & $1.6(1.2,2.3)$ & $1.6(1.2,2.3)$ & $1.6(1.2,2.3)$ & $1.6(1.2,2.3)$ \\
\hline \multicolumn{6}{|l|}{ History of complications, n (\%) } \\
\hline Coronary arterial disease & $2380(21)$ & 1694 & $686(29)$ & 1611 & $769(32)$ \\
\hline Cerebrovascular disease & $1439(13)$ & 1107 & $332(23)$ & 1099 & $340(24)$ \\
\hline Diabetic kidney disease & $2444(22)$ & 1771 & $673[28]$ & 1699 & $745(30)$ \\
\hline Diabetic retinopathy & $1079(10)$ & 812 & $267(25)$ & 748 & $331(31)$ \\
\hline Dementia & $109(1)$ & 75 & $34(31)$ & 67 & $42(39)$ \\
\hline \multicolumn{6}{|l|}{ History of tobacco smoking, n (\%) } \\
\hline Ever & $4674(42)$ & 3303 & $1371(29)$ & 3216 & $1458(31)$ \\
\hline Current & $1682(15)$ & 1287 & $395(23)$ & 1212 & $470(28)$ \\
\hline \multicolumn{6}{|l|}{ History of alcohol drinking, n (\%) } \\
\hline Past & $4237(38)$ & 3015 & $1222(29)$ & 2997 & $1240(29)$ \\
\hline Current & $3396(30)$ & 2433 & $963(28)$ & 2368 & $1028(30)$ \\
\hline \multicolumn{6}{|l|}{ History of medication use, n (\%) } \\
\hline Metformin & $6752(61)$ & 5238 & $1514(22)$ & 4836 & $1916(28)$ \\
\hline Insulin therapy & $159(1)$ & 133 & $26(16)$ & 118 & $41(26)$ \\
\hline Antihypertensive treatment & 7655 (69) & 5908 & $1747(23)$ & 5552 & $2103(27)$ \\
\hline
\end{tabular}


Table 1 (continued)

\begin{tabular}{|c|c|c|c|c|c|}
\hline & \multirow[t]{2}{*}{ Overall } & \multicolumn{2}{|c|}{ Peripheral artery disease } & \multicolumn{2}{|c|}{ Peripheral neuropathy } \\
\hline & & No & Yes & No & Yes \\
\hline Antiplatelet drugs & $5199(47)$ & 3914 & $1285[25]$ & 3694 & $1505(29)$ \\
\hline Statins & $3146(28)$ & 2207 & $939(30)$ & 2121 & 1025 [33] \\
\hline \multicolumn{6}{|l|}{ Study allocations, n (\%) } \\
\hline Intensive glucose control group & $5571(50)$ & 4334 & $1237(22)$ & 4063 & $1508(27)$ \\
\hline Perindopril/indapamide combination & $5569(50)$ & 4365 & $1204(22)$ & 4070 & $1499(27)$ \\
\hline
\end{tabular}

Categorical parameters are expressed as numbers (and percentages for participants with baseline history of PAD or peripheral neuropathy). ACR albumin to creatinine ratio. eGFR estimated Glomerular Filtration Rate computed by the Chronic Kidney Disease Epidemiology Collaboration equation. Mini-Mental State Examination (MMSE) score: normal ( $\geq 28)$, reduced $(<28)$. Education accomplishment (age at completion of the highest level of formal education): basic $(\geq 16$ years), low $(\leq 15$ years)

\section{Incidence of cancer death}

Cancer death occurred in $316(2.8 \%)$ participants during a median of $5.0\left(25^{\text {th }}-75^{\text {th }}\right.$ percentile, $\left.4.7-5.1\right)$ years of follow-up corresponding to 53,550 person-years and an incidence rate of 5.9 (95\% CI 5.3-6.6) per 1000 person-years. The cumulative incidence of cancer death was $2.1 \%$ in women and $3.4 \%$ in men. The baseline mean (SD) age, duration of diabetes, HbA1c, systolic and diastolic blood pressure of individuals who subsequently died from cancer causes were 69 (6) years, 7.9 (6.6) years, 7.6 (6.6\%), 146 (22) $\mathrm{mmHg}$ and 80 (11) $\mathrm{mmHg}$, respectively (Table 2). Hazard ratio for cancer death according to each individual parameter is shown in Table 3, after adjusting for study allocations.

\section{Effects of study treatments on the risk for cancer death}

Cancer death occurred in 147 (2.6\%) participants assigned to perindopril/indapamide combination versus 169 (3.0\%) individuals assigned to placebo (incidence rate: 5.5 [95\% CI, 4.6-6.4] versus 6.3 [5.4-7.4] per 1000 person-years) with no significant difference between blood pressure treatment groups: HR 0.86 (95\% CI, 0.69-1.08). Cancer death occurred in 65 (2.3\%) participants [incidence rate $4.8(3.8-6.2)$ per 1000 personyears] randomized to both intensive glucose control and active blood pressure treatment versus 86 (3.1\%) individuals [incidence rate 6.4 (5.2-8.0) per 1000 person-years] assigned to standard glucose control and placebo group [HR $0.75(0.54-1.03)]$.

\section{Risk of cancer death according to history of lower-limb complications at baseline}

The cumulative incidences (Table 4 and Fig. 1) and incidence rates of cancer death were higher in participants with a baseline history of lower-limb complications [ 8.3 (95\% CI, 7.2-9.7] vs. 4.4 (3.7-5.2) per 1000 person-years], PAD [8.9 (7.3-10.8) vs. 5.1 (4.4-5.8) per 1000 personyears] or peripheral neuropathy [8.4 (7.1-10.1) vs. 5.0
[4.3-5.7] per 1000 person-years), compared with individuals without this complication. The risk of cancer death was higher in patients with a baseline history of lowerlimb complication [HR $1.62(1.29-2.04), p<0.0001]$, PAD [1.43 (1.12-1.83), $p=0.004]$ or peripheral neuropathy [1.49 (1.19-1.88), $p=0.0006]$, compared with those without these conditions after adjusting for age (and its square), sex, region of origin and study allocations. These associations remained significant after further adjustment for a wide range of potential confounders (model 2, Table 4). When we used our alternative definition of lower-limb complication, PAD and peripheral neuropathy remained associated with increased risk of cancer death, but not the history of tissue or limb loss (Additional file 1: Table S2). Comparable results were also observed when peripheral neuropathy was defined as the presence of at least one neurological abnormality or a history of foot ulceration (Additional file 1: Table S3). Associations between lower-limb complications and risk of cancer death were comparable when were stratified analyses by smoking status, with no evidence for statistical interaction (Additional file 1: Table S4). No statistical interaction was observed between PAD and peripheral neuropathy in their association with cancer death $(p=0.14)$.

\section{Risk of cancer death according to baseline history of lower-limb complications considering death from non-cancer causes as a competing risk}

All-cause and cardiovascular death occurred in 1031 (9.2\%) and 542 (4.9\%) participants during follow-up. Their incidence rates were 19.2 (95\% CI 18.1-20.5) and $10.1(9.3-11.0)$ per 1000 person-years, respectively. We observed an increased risk of all-cause and cardiovascular death in patients with a baseline history of lower-limb complications or PAD, compared with those without these complications (Additional file 1: Table S5). The history of peripheral neuropathy was associated with 
Table 2 Characteristics of participants at baseline according to the incidence of cancer death during follow-up

\begin{tabular}{|c|c|c|}
\hline & \multicolumn{2}{|c|}{ Cancer death } \\
\hline & No & Yes \\
\hline N & 10,824 & $316(2.8)$ \\
\hline \multicolumn{3}{|l|}{ Gender, n (\%) } \\
\hline Women & 4633 & $100(2.1)$ \\
\hline Men & 6191 & $216(3.4)$ \\
\hline \multicolumn{3}{|l|}{ Region of origin, $n(\%)$} \\
\hline Asia & 4059 & $77(1.9)$ \\
\hline Established market economies & 4688 & $174(3.6)$ \\
\hline Eastern Europe & 2077 & $65(3.0)$ \\
\hline Age, years: mean (SD) & $66(6)$ & $69(6)$ \\
\hline Duration of diabetes, years: mean (SD) & $7.9(6.3)$ & $7.9(6.6)$ \\
\hline Body mass index, $\mathrm{kg} / \mathrm{m}^{2}$ : mean (SD) & $28(5)$ & $29(5)$ \\
\hline Waist circumference, cm: mean (SD) & $98(13)$ & $101(13)$ \\
\hline Systolic blood pressure, mmHg: mean (SD) & $145(21)$ & $146(22)$ \\
\hline Diastolic blood pressure, mmHg: mean (SD) & $81[11]$ & $80[11]$ \\
\hline \multicolumn{3}{|l|}{ Mini-mental state examination } \\
\hline Overall MMSE score, mean (SD) & $28(2)$ & $28(2)$ \\
\hline Participants with normal MMSE score, n (\%) & 8456 & $233(2.7)$ \\
\hline Participants with reduced MMSE score, n (\%) & 2368 & $83(3.4)$ \\
\hline \multicolumn{3}{|l|}{ Education accomplishment } \\
\hline Age at completion, years: mean (SD) & $18(7)$ & $17(6)$ \\
\hline Participants with basic education, n (\%) & 6941 & $175(2.5)$ \\
\hline Participants with low education, n (\%) & 3883 & $141(3.5)$ \\
\hline \multicolumn{3}{|l|}{ HbA1c, mean (SD) } \\
\hline$\%$ & $7.5(1.5)$ & $7.6(1.6)$ \\
\hline $\mathrm{mmol} / \mathrm{mol}$ & $59(17)$ & $59(17)$ \\
\hline Urinary ACR, $\mu \mathrm{g} / \mathrm{mg}$ : median (25th, 75th percentiles) & $1(1,2)$ & $1(1,2)$ \\
\hline eGFR, ml/min/1.73m²: mean (SD) & $74(17)$ & $71(17)$ \\
\hline Serum total cholesterol, mmol/l: mean (SD) & $5.2(1.2)$ & $5.0(1.1)$ \\
\hline Serum HDL cholesterol, mmol/l: mean (SD) & $1.2(0.3)$ & $1.2(0.3)$ \\
\hline Serum LDL cholesterol, mmol/l: mean (SD) & $3.1(1.0)$ & $2.9(1.0)$ \\
\hline Serum triglycerides, $\mathrm{mmol} / \mathrm{l}$ : median (25th, 75 th percentiles) & $1.6(1.2,2.3)$ & $1.6(1.1,2.3)$ \\
\hline \multicolumn{3}{|l|}{ History of complications, n (\%) } \\
\hline Coronary arterial disease & 2318 & $62(2.6)$ \\
\hline Cerebrovascular disease & 1401 & $38(2.6)$ \\
\hline Diabetic kidney disease & 2358 & $86(3.5)$ \\
\hline Diabetic retinopathy & 1041 & $38(3.5)$ \\
\hline Dementia & 107 & $2(1.8)$ \\
\hline \multicolumn{3}{|l|}{ History of tobacco smoking, n (\%) } \\
\hline Ever & 4501 & $173(3.7)$ \\
\hline Current & 1626 & $56(3.3)$ \\
\hline \multicolumn{3}{|l|}{ History of alcohol drinking, n (\%) } \\
\hline Past & 4097 & $140(3.3)$ \\
\hline Current & 3281 & $115(3.4)$ \\
\hline \multicolumn{3}{|l|}{ History of medication use, n (\%) } \\
\hline Metformin & 6582 & $170(2.5)$ \\
\hline Insulin therapy & 154 & $5(3.1)$ \\
\hline Antihypertensive treatment & 7448 & $207(2.7)$ \\
\hline Antiplatelet drugs & 5058 & $141(2.7)$ \\
\hline
\end{tabular}


Table 2 (continued)

\begin{tabular}{lll}
\hline & Cancer death & Yes \\
\cline { 3 - 3 } & No & $88(2.8)$ \\
Statins & 3058 & $148(2.7)$ \\
Study allocations, $n(\%)$ & & $147(2.6)$ \\
Intensive glucose control group & 5423 & 5422 \\
Perindopril/indapamide combination & & 147 \\
\hline
\end{tabular}

Categorical parameters are expressed as numbers (and percentages for participants who died from cancer causes)

ACR albumin to creatinine ratio. eGFR estimated Glomerular Filtration Rate computed by the Chronic Kidney Disease Epidemiology Collaboration equation. MiniMental State Examination (MMSE) score: normal $(\geq 28)$, reduced $(<28)$. Education accomplishment (age at completion of the highest level of formal education): basic $(\geq 16$ years), low $(\leq 15$ years)

Table 3 Risk of cancer death according to each individual parameter at baseline

\begin{tabular}{|c|c|c|c|}
\hline & Hazard Ratio & $95 \% \mathrm{Cl}$ & $p$ \\
\hline Female sex & 0.62 & $0.49-0.79$ & $<0.0001$ \\
\hline Region of origin: established market economies (vs. Asia) & 1.87 & $1.43-2.44$ & $<0.0001$ \\
\hline Region of origin: Eastern Europe (vs. Asia) & 1.68 & $1.21-2.33$ & 0.002 \\
\hline Region of origin: established market economies (vs. Eastern Europe) & 1.11 & $0.84-1.48$ & 0.46 \\
\hline Age (per 1 SD increase) & 1.82 & $1.63-2.03$ & $<0.0001$ \\
\hline Duration of diabetes (per 1 SD increase) & 1.01 & $0.91-1.13$ & 0.83 \\
\hline Body mass index (per 1 SD increase) & 1.08 & $0.97-1.20$ & 0.14 \\
\hline Waist circumference (per 1 SD increase) & 1.23 & $1.11-1.37$ & $<0.0001$ \\
\hline Systolic blood pressure (per 1 SD increase) & 1.07 & $0.96-1.19$ & 0.23 \\
\hline Diastolic blood pressure (per 1 SD increase) & 0.91 & $0.81-1.02$ & 0.10 \\
\hline Mini-Mental State Examination score (<28 vs. $\geq 28)$ & 1.30 & $1.01-1.67$ & 0.04 \\
\hline Education accomplishment ( $\leq 15 \mathrm{vs} . \geq 16$ years) & 1.44 & $1.15-1.80$ & 0.001 \\
\hline HbA1c (per 1 SD increase) & 1.04 & $0.93-1.16$ & 0.45 \\
\hline Urinary albumin to creatinine ratio (per 1SD increase) & 1.08 & $0.97-1.21$ & 0.16 \\
\hline Estimated glomerular filtration rate (per 1 SD increase) & 0.82 & $0.73-0.91$ & 0.0003 \\
\hline Serum total cholesterol (per 1 SD increase) & 0.85 & $0.75-0.95$ & 0.007 \\
\hline Serum HDL cholesterol (per 1 SD increase) & 0.88 & $0.78-0.99$ & 0.03 \\
\hline Serum LDL cholesterol (per 1 SD increase) & 0.84 & $0.75-0.95$ & 0.004 \\
\hline Serum triglycerides (per 1 SD increase) & 0.97 & $0.86-1.09$ & 0.60 \\
\hline History of coronary arterial disease & 0.91 & $0.69-1.20$ & 0.52 \\
\hline History of cerebrovascular disease & 0.96 & $0.68-1.34$ & 0.79 \\
\hline History of diabetic kidney disease & 1.38 & $1.08-1.77$ & 0.01 \\
\hline History of diabetic retinopathy & 1.34 & $0.96-1.89$ & 0.09 \\
\hline History of dementia & 0.63 & $0.16-2.52$ & 0.51 \\
\hline History of current smoking & 1.21 & $0.90-1.61$ & 0.20 \\
\hline History of ever smoking & 1.67 & $1.33-2.08$ & $<0.0001$ \\
\hline History of past alcohol drinking & 1.28 & $1.02-1.60$ & 0.03 \\
\hline History of current alcohol drinking & 1.27 & $1.01-1.60$ & 0.04 \\
\hline Use of Metformin & 0.75 & $0.60-0.93$ & 0.01 \\
\hline Use of insulin therapy & 1.17 & $0.48-2.83$ & 0.72 \\
\hline Use of antihypertensive treatment & 0.88 & $0.70-1.11$ & 0.27 \\
\hline Use of antiplatelet drugs & 0.92 & $0.74-1.15$ & 0.48 \\
\hline Use of statins & 0.95 & $0.75-1.22$ & 0.71 \\
\hline
\end{tabular}

Cox proportional hazards survival regressive analysis for each variable adjusted for study allocations. SD standard deviation 
Table 4 Risk of cancer death according to history of lower-limb complications at baseline

\begin{tabular}{|c|c|c|c|c|c|c|c|}
\hline & & \multicolumn{2}{|c|}{ Cancer death, n (\%) } & \multicolumn{2}{|l|}{ Model 1} & \multicolumn{2}{|l|}{ Model 2} \\
\hline & & No, $n$ & Yes, n (\%) & Hazard ratio $(95 \% \mathrm{Cl})$ & $p$ & Hazard ratio $(95 \% \mathrm{Cl})$ & $p$ \\
\hline \multirow[t]{2}{*}{ Lower-limb complication } & No & 6702 & $145(2.1)$ & $1.62(1.29-2.04)$ & $<0.0001$ & $1.53(1.21-1.94)$ & 0.0004 \\
\hline & Yes & 4122 & $171(4.0)$ & & & & \\
\hline \multirow[t]{2}{*}{ Peripheral arterial disease } & No & 8488 & $213(2.4)$ & $1.43(1.12-1.83)$ & 0.004 & $1.32(1.02-1.70)$ & 0.03 \\
\hline & Yes & 2336 & $103(4.2)$ & & & & \\
\hline \multirow[t]{2}{*}{ Peripheral neuropathy } & No & 7971 & $196(2.4)$ & $1.49(1.19-1.88)$ & 0.0006 & $1.41(1.11-1.79)$ & 0.004 \\
\hline & Yes & 2853 & $120(4.0)$ & & & & \\
\hline
\end{tabular}

Model 1: age (and its square), sex, region of origin, and study allocations

Model 2: model 1 plus duration of diabetes, body mass index (and its square), waist circumference (and its square), systolic and diastolic blood pressure (and their squares), HbA1c (and its square), urinary ACR, eGFR (and its square), total cholesterol, HDL cholesterol, triglycerides, MMSE score, education accomplishment, history of ever or current smoking, history of past or current alcohol drinking, history of CAD, cerebrovascular disease, diabetic retinopathy or dementia, and use of metformin, insulin, antihypertensive, statin or antiplatelet therapy

increased risk of all-cause, but not cardiovascular, death (Additional file 1: Table S5). The association between lower-limb complications and cancer death did not change substantially when we considered death from non-cancer causes as a competing risk (Additional file 1: Table S5).

\section{Risk of incident cancers according to history of lower-limb complications at baseline}

Incident cancers were diagnosed in 700 (6.3\%) participants during follow-up (Additional file 1: Table S1), corresponding to 52,473 person-years and an incidence rate of 13.3 (95\% CI, 12.4-14.4) per 1000 person-years. The cumulative incidences (Table 5) and incidence rates of cancers were higher in participants with a baseline history of lower-limb complication [17.1 (95\% CI, 15.419.0) vs.11.0 (9.9-12.2) per 1000 person-years], PAD [18.9 (16.5-21.6) vs. $11.8(10.8-12.9)$ per 1000 personyears] or peripheral neuropathy $[16.7$ (14.7-19.0) vs. 12.1 (11.1-13.3) per 1000 person-years], compared with those without these conditions. The relative risk of incident cancers was higher in participants with (vs. without) a baseline history of lower-limb complications [HR $1.23(1.05-1.44), p=0.01]$ or PAD (1.20 (1.01-1.43), $p=0.04]$, but not those with peripheral neuropathy $[1.14$ (0.97-1.35), $p=0.11$ ] after adjustment for cofounding variables as in model 2 (Table 5). Association with PAD was particularly observed for solid and digestive malignant neoplasms (Additional file 1: Table S6). Based on the alternative definitions of lower-limb complications, PAD remained associated with excess risk of incident cancers, while peripheral neuropathy and tissue or limb loss did not (Additional file 1: Table S2). The history of diabetic neuropathy, defined as the presence of at least one neurological abnormality or a history of foot ulceration, was not associated with the risk of incident cancers (Additional file 1: Table S3).

\section{Discussion}

In the present study, we investigated the relationship between a baseline history of lower-limb complications and the risk of cancer death in patients with type 2 diabetes in the ADVANCE study. We observed an independent association between the history of lower-limb complications, both PAD and peripheral neuropathy, and excess 5-year risk of cancer death. No statistical interaction was observed between PAD and sensory peripheral neuropathy in their association with the risk of cancer death. Our findings were reliable when we considered death from non-cancer causes as a competing risk. Of note, lower-limb complications and PAD were associated with increased risk of both all-cause and cardiovascular death, while neuropathy was associated with only allcause death. The history of PAD, but not peripheral neuropathy, was also associated with a higher incidence of cancers, mainly solid and digestive neoplasms.

\section{Lower-limb complications and risk of cancer death}

As far as we know, this is the first report of increased risk of cancer death related to lower-limb complications in patients with type 2 diabetes. Few previous studies reported increased incidence of cancers among patients with intermittent claudication or critical limb ischemia in general population [30, 31]. A recent study has shown an association between lower-limb arterial thrombosis and increased risk of subsequent cancers in 6600 patients (12\% with diabetes) from Danish nationwide populationbased registries [32]. A lower-limb arterial thrombosis was also associated with a high risk of all-cause mortality following some site-specific (mainly smoking-dependent) cancers. 


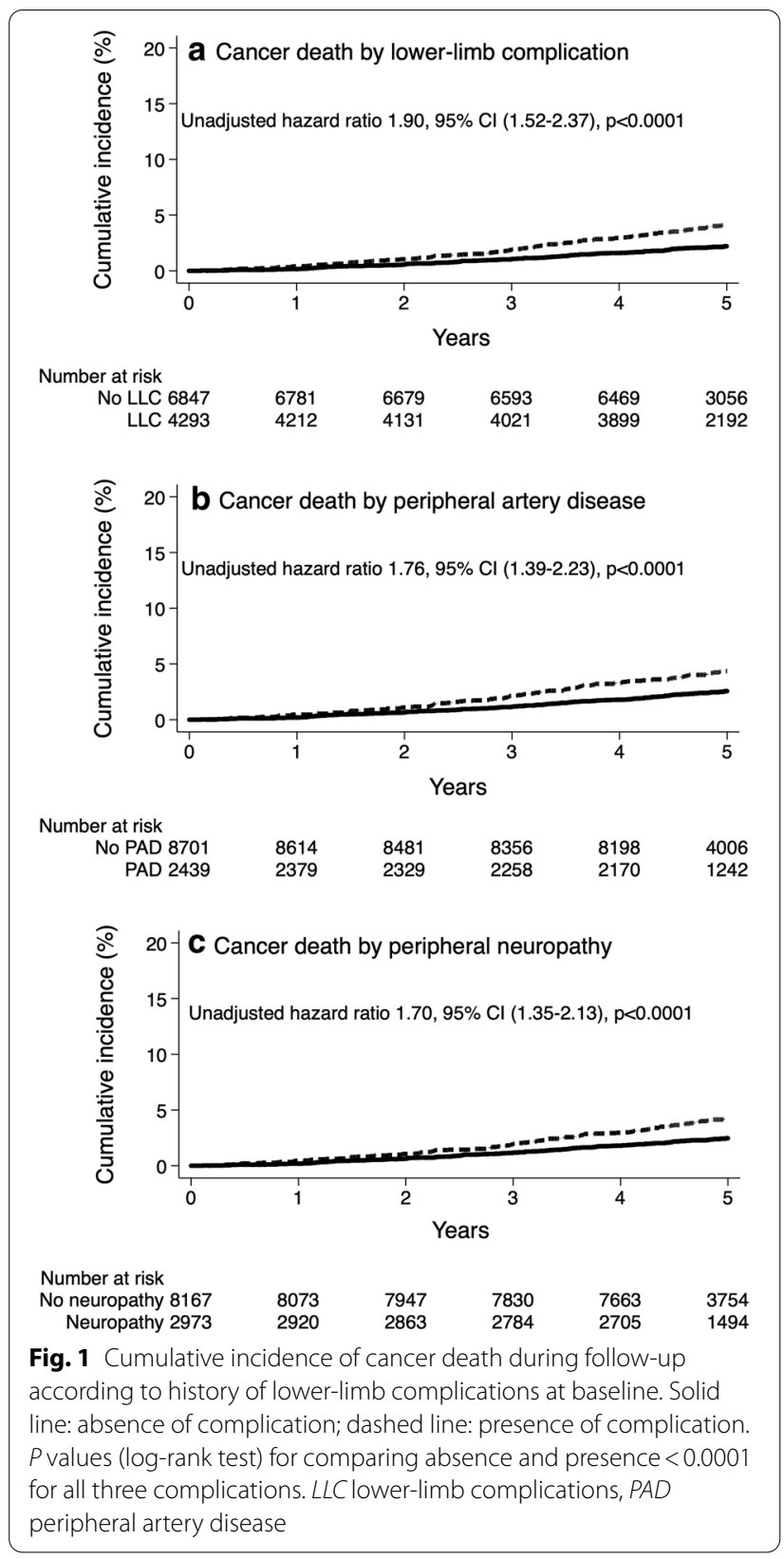

\section{Potential mechanisms linking lower-limb complications to cancer death}

Our findings cannot allow any etiological conclusion, and may only suggest lower-limb complications as proxy for cancer death. Indeed, lower-limb complications are surrogates for advanced microvascular and macrovascular disease, and may lead to a greater likelihood of multisystem disease. Nevertheless, lower-limb complications and malignancies may share some modifiable risk factors, including age, obesity and tobacco smoking [33-35]. These risk factors are unlikely to explain our findings as the key observed associations were reliable after adjustment for these confounders. Furthermore, the magnitude of the associations between lower-limb complications and cancer death were comparable when we performed analyses in people who have smoked cigarettes regularly and those who have never smoked, without evidence for statistical interaction. Further studies are needed to investigate the potential contribution of smoking in the relationship between lower-limb complications and cancer death. Lower-limb complications and malignancies may also share some common biological disorders including chronic inflammation, increased advanced glycation endproducts and oxidative stress [36-39]. Oxidative stress leads to lipid peroxidation and DNA damage, which are involved in microvascular disease, atherosclerosis and cancer [36, 40]. Furthermore, endothelial dysfunction, an important impairment involved in lower-limb complications, has also been linked to increased risk of solid-tumor cancer [41]. While lower-limb complications, involving microvascular disease and atherosclerosis (as a result at least partly of early endothelial dysfunction), cancer is mainly characterized by excessive angiogenesis, which may be driven by endothelial metabolic aberrations [42].

\section{Effects of glucose and blood pressure controls in risk of cancer death}

We did not observe an association between ADVANCE allocation to blood pressure treatment and the risk of cancer death, as was previously reported for intensive glucose control [28]. However, we have observed a nonsignificant decrease in risk of cancer death in participants assigned to both glucose control and blood pressure interventions, compared with those allocated to conventional glucose control and placebo blood pressure treatments. This observation may raise the hypothesis wondering the potential effect of multifactorial intervention on the incidence of cancer death in people with type 2 diabetes. Randomised clinical trials are required to address this hypothesis. Of note, Rasmussen-Torvik and coworkers reported an inverse association between the number of ideal cardiovascular health metrics, as defined by the American Heart Association [43] (including blood pressure and glycemia) and combined cancer incidence in the Atherosclerosis Risk In Communities (ARIC) cohort [44].

\section{Strengths and limitations}

The main strength of our work is the use of a large international multicentre study of 11,140 patients with type 2 diabetes, with a comprehensive range of data including demographic, clinical and biological features at baseline. Participants were also prospectively followed 
Table 5 Risk of incident cancers according to history of lower-limb complications at baseline

\begin{tabular}{|c|c|c|c|c|c|c|c|}
\hline & & \multicolumn{2}{|c|}{ Incident cancers, n (\%) } & \multicolumn{2}{|l|}{ Model 1} & \multicolumn{2}{|l|}{ Model 2} \\
\hline & & No, $n$ & Yes, n (\%) & Hazard ratio $(95 \% \mathrm{Cl})$ & $p$ & Hazard ratio $(95 \% \mathrm{Cl})$ & $p$ \\
\hline \multirow[t]{2}{*}{ Lower-limb complication } & No & 6490 & $357(5.2)$ & $1.34(1.15-1.56)$ & 0.0001 & $1.23(1.05-1.44)$ & 0.01 \\
\hline & Yes & 3950 & $343(8.0)$ & & & & \\
\hline \multirow[t]{2}{*}{ Peripheral arterial disease } & No & 8214 & $487(5.6)$ & $1.32(1.12-1.56)$ & 0.001 & $1.20(1.01-1.43)$ & 0.04 \\
\hline & Yes & 2226 & $213(8.7)$ & & & & \\
\hline \multirow[t]{2}{*}{ Peripheral neuropathy } & No & 7699 & $468(5.7)$ & $1.24(1.06-1.45)$ & 0.008 & $1.14(0.97-1.35)$ & 0.11 \\
\hline & Yes & 2741 & $232(7.8)$ & & & & \\
\hline
\end{tabular}

Model 1: age (and its square), sex, region of origin, and study allocations

Model 2: model 1 plus duration of diabetes, body mass index (and its square), waist circumference (and its square), systolic and diastolic blood pressure (and their squares), HbA1c (and its square), urinary ACR, eGFR (and its square), total cholesterol, HDL cholesterol, triglycerides, MMSE score, education accomplishment, history of ever or current smoking, history of past or current alcohol drinking, history of CAD, cerebrovascular disease, diabetic retinopathy or dementia, and use of metformin, insulin, antihypertensive, statin or antiplatelet therapy

for 5 years, with adjudicated causes of mortality, including cancer death within a central and independent adjudication committee process. However, incident cancers were collected from case report forms and reports of serious adverse events, without adjudication or validation using cancer registry data. The other limitation of our study is related to the post hoc analyses of a randomized controlled trial, and the use of a clinical trial population, which may not be representative of all patients with type 2 diabetes. Also, we used a pragmatic assessment of lower-limb complications without a prespecified research protocol, and we missed some data regarding PAD (intermittent claudication, ankle brachial index, toe pressure or transcutaneous oxygen pressure) or peripheral sensory neuropathy (symptoms or vibration sensation test). We do not believe that these issues influenced our findings as we investigated a widespread spectrum of data including clinical examination (pulses palpation, light touch sensation and reflex tests), condition (foot ulceration) and procedures (revascularisation and lower-extremity amputation). However, we cannot exclude that non-diabetic causes of neuropathy could have biased our findings, especially neurotoxicity of cancer treatment (chemotherapeutic drugs and radiotherapy), paraneoplastic neurologic disorders or nervous dysfunction caused by the cancer [45]. Nevertheless, cancer-related neuropathy seemed to be unlikely, since we dealt with a high prevalent neuropathy $(n=2973,27 \%)$ consistent with diabetic origin, while incident cancers were diagnosed only in $700(6 \%)$ participants. Finally, our findings were not controlled for socioeconomic position as we missed appropriate data, except for education accomplishment.

\section{Conclusions}

In summary, our study links PAD and sensory peripheral neuropathy to excess 5-year risk for cancer death in patients with type 2 diabetes. In addition, PAD was also associated with increased risk of incident cancers, especially solid and digestive ones. Our findings provide new evidence on the non-cardiovascular prognostic burden of lower-limb complications in people with type 2 diabetes, and encourage continuing careful evaluation and management of cancers in this population.

\section{Supplementary Information}

The online version contains supplementary material available at https://doi. org/10.1186/s12933-020-01198-y.

Additional file 1: Table S1. Site-specific cancers and their frequencies in the ADVANCE study. Table S2. Risk of cancer death and incident cancers according to history of lower-limb complications (using an alternative definition) at baseline. Table S3. Risk of cancer death and incident cancers according to history of diabetic neuropathy (using an alternative definition) at baseline. Table S4. Risk of cancer death according to history of lower-limb complications by smoking status at baseline. Table S5. Risks of cardiovascular, all-cause and cancer (considering death from non-cancer causes as a competing risk) death according to history of lower-limb complications at baseline. Table S6. Risk of site-specific cancers according to history of lower-limb complications at baseline

\section{Abbreviations}

ADVANCE: Action in Diabetes and Vascular Disease: PreterAx and DiamicroN Modified-Release Controlled Evaluation; ACR: Albumin-to-creatinine ratio; ARIC: Atherosclerosis Risk In Communities cohort; Cl: Confidence interval; DKD: Diabetic Kidney Disease; eGFR: Estimated Glomerular Filtration Rate; HR: Hazard ratio; ICD-10: International Classification of Diseases Code Tenth Revision; MMSE: Mini-Mental State Examination; PAD: Peripheral Artery Disease; SD: Standard Deviation. 


\section{Acknowledgements}

KM was supported by grants from the Société Francophone du Diabète (SFD) and the Association Diabète Risque Vasculaire (ADRV), and travel support from the Association pour le Développement de l'Enseignement et des Recherches auprès des universités, des centres de recherches et des entreprises d'Aquitaine (ADERA).

\section{Authors' contributions}

$\mathrm{KM}, \mathrm{JC}$ and $\mathrm{MW}$ designed the study. KM drafted the manuscript. SH, GM, MM NP, JC and MW researched and interpreted data, contributed to discussion and reviewed/edited the manuscript. All authors approved the current version of the manuscript. KM is the guarantor of this work and, as such, had full access to all the data in the study and take responsibility for the integrity of the data and the accuracy of the data analyses. All authors read and approved the final manuscript.

\section{Funding}

The ADVANCE trial was funded by grants from the National Health and Medical Research Council (NHMRC) of Australia and Servier. MW is supported by grants 1080206 and 1149987 from the National Health and Medical Research Council (NHMRC) of Australia.

\section{Availability of data and materials}

The datasets analysed during the current study are not publicly available due to consideration of intellectual property, due to many ongoing active collaborations worldwide, and to continuing analyses by the study investigators, but may be available from the principal investigator on reasonable request.

\section{Ethics approval and consent to participate}

The Institutional Ethics Committee of each participating centre approved the ADVANCE study, and all participants provided written informed consent.

\section{Consent for publication}

Not applicable.

\section{Competing interests}

MW is a consultant to Amgen and Kyowa Kirin. Authors declare no other potential conflict of interest relevant to this article.

\section{Author details}

${ }^{1}$ Department of Endocrinology, Diabetes and Nutrition, Bordeaux University Hospital, Bordeaux, France. ${ }^{2}$ Faculty of Medicine, The University of Bordeaux, Bordeaux, France. ${ }^{3}$ INSERM Unit 1034, Bordeaux, France. ${ }^{4}$ The University of Melbourne and Royal Melbourne Hospital, Melbourne, VIC, Australia. ${ }^{5}$ The University of Milan-Bicocca and Istituto Auxologico Italiano, Milan, Italy. ${ }^{6}$ Université de Paris, UFR de Médecine, Paris, France. ${ }^{7}$ Centre de Recherche Des Cordeliers, INSERM, Sorbonne Université, Université de Paris, Paris, France. ${ }^{8}$ CMC Ambroise Paré, Neuilly-sur-Seine, France. ${ }^{9}$ The International Centre for Circulatory Health, National Heart and Lung Institute, Imperial College, London, UK. ${ }^{10}$ The George Institute for Global Health, Sydney, NSW, Australia. ${ }^{11}$ The University of New South Wales, Sydney, NSW, Australia. ${ }^{12}$ Faculty of Medicine, Imperial College London, London, UK. ${ }^{13}$ Department of Epidemiology, Johns Hopkins University, Baltimore, MD, USA.

Received: 26 October 2020 Accepted: 14 December 2020 Published online: 04 January 2021

\section{References}

1. Boulton AJ, Vileikyte L, Ragnarson-Tennvall G, Apelqvist J. The global burden of diabetic foot disease. Lancet. 2005;366(9498):1719-24.

2. Armstrong DG, Boulton AJM, Bus SA. Diabetic foot ulcers and their recurrence. N Engl J Med. 2017;376(24):2367-75.

3. Nativel M, Potier L, Alexandre L, Baillet-Blanco L, Ducasse E, Velho G, et al. Lower extremity arterial disease in patients with diabetes: a contemporary narrative review. Cardiovasc Diabetol. 2018;17(1):138.

4. Feldman EL, Callaghan BC, Pop-Busui R, Zochodne DW, Wright DE, Bennett DL, et al. Diabetic neuropathy. Nat Rev Dis Primers. 2019;5(1):41.
5. Geiss LS, Li Y, Hora I, Albright A, Rolka D, Gregg EW. Resurgence of diabetes-related nontraumatic lower-extremity amputation in the young and middle-aged adult U.S. population. Diabetes Care. 2019;42(1):50-4.

6. Johannesson A, Larsson GU, Ramstrand N, Turkiewicz A, Wirehn AB, Atroshi I. Incidence of lower-limb amputation in the diabetic and nondiabetic general population: a 10-year population-based cohort study of initial unilateral and contralateral amputations and reamputations. Diabetes Care. 2009;32(2):275-80.

7. Buckley CM, O'Farrell A, Canavan RJ, Lynch AD, De La Harpe DV, Bradley $C P$, et al. Trends in the incidence of lower extremity amputations in people with and without diabetes over a five-year period in the Republic of Ireland. PLoS ONE. 2012;7(7):e41492.

8. Kerr M, Barron E, Chadwick P, Evans T, Kong WM, Rayman G, et al. The cost of diabetic foot ulcers and amputations to the National Health Service in England. Diabet Med. 2019;36(8):995-1002.

9. Zhang Y, Lazzarini PA, McPhail SM, van Netten JJ, Armstrong DG, Pacella RE. Global disability burdens of diabetes-related lower-extremity complications in 1990 and 2016. Diabetes Care. 2020;43(5):964-74.

10. Walsh JW, Hoffstad OJ, Sullivan MO, Margolis DJ. Association of diabetic foot ulcer and death in a population-based cohort from the United Kingdom. Diabet Med. 2016;33(11):1493-8.

11. Mohammedi K, Woodward M, Hirakawa Y, Zoungas S, Colagiuri S, Hamet $P$, et al. Presentations of major peripheral arterial disease and risk of major outcomes in patients with type 2 diabetes: results from the ADVANCE-ON study. Cardiovasc Diabetol. 2016;15(1):129.

12. Mohammedi K, Woodward M, Zoungas S, Li Q, Harrap S, Patel A, et al. Absence of peripheral pulses and risk of major vascular outcomes in patients with type 2 diabetes. Diabetes Care. 2016;39(12):2270-7.

13. Bjerg L, Hulman A, Carstensen B, Charles M, Witte DR, Jorgensen ME. Effect of duration and burden of microvascular complications on mortality rate in type 1 diabetes: an observational clinical cohort study. Diabetologia. 2019;62(4):633-43.

14. Brownrigg JR, Griffin M, Hughes CO, Jones KG, Patel N, Thompson MM, et al. Influence of foot ulceration on cause-specific mortality in patients with diabetes mellitus. J Vasc Surg. 2014;60(4):982-6.e3.

15. Lozano R, Naghavi M, Foreman K, Lim S, Shibuya K, Aboyans V, et al. Global and regional mortality from 235 causes of death for 20 age groups in 1990 and 2010: a systematic analysis for the Global Burden of Disease Study 2010. Lancet. 2012;380(9859):2095-128.

16. Seshasai SR, Kaptoge S, Thompson A, Di Angelantonio E, Gao P, et al. Diabetes mellitus, fasting glucose, and risk of cause-specific death. N Engl J Med. 2011;364(9):829-41.

17. Campbell PT, Newton CC, Patel AV, Jacobs EJ, Gapstur SM. Diabetes and cause-specific mortality in a prospective cohort of one million U.S. adults. Diabetes Care. 2012;35(9):1835-44.

18. Harding JL, Andes $\sqcup$, Gregg EW, Cheng YJ, Weir HK, Bullard KM, et al. Trends in cancer mortality among people with vs without diabetes in the USA, 1988-2015. Diabetologia. 2020;63(1):75-84.

19. Ling S, Brown K, Miksza JK, Howells LM, Morrison A, Issa E, et al. Risk of cancer incidence and mortality associated with diabetes: A systematic review with trend analysis of 203 cohorts. Nutr Metab Cardiovasc Dis. 2020. https://doi.org/10.1016/j.numecd.2020.09.023.

20. Youn JC, Chung WB, Ezekowitz JA, Hong JH, Nam H, Kyoung DS, et al. Cardiovascular disease burden in adult patients with cancer: An 11-year nationwide population-based cohort study. Int J Cardiol. 2020;317:167-73

21. Wong G, Zoungas S, Lo S, Chalmers J, Cass A, Neal B, et al. The risk of cancer in people with diabetes and chronic kidney disease. Nephrol Dial Transplant. 2012;27(8):3337-44.

22. Yu TY, Li HY, Jiang YD, Chang TJ, Wei JN, Chuang LM. Proteinuria predicts 10-year cancer-related mortality in patients with type 2 diabetes. J Diabetes Complications. 2013;27(3):201-7.

23. Bendor CD, Bardugo A, Pinhas-Hamiel O, Afek A, Twig G. Cardiovascular morbidity, diabetes and cancer risk among children and adolescents with severe obesity. Cardiovasc Diabetol. 2020;19(1):79.

24. ADVANCE Management Committee. Study rationale and design of ADVANCE: action in diabetes and vascular disease-preterax and diamicron MR controlled evaluation. Diabetologia. 2001;44(9):1118-20.

25. Patel A, MacMahon S, Chalmers J, Neal B, Woodward M, et al. Effects of a fixed combination of perindopril and indapamide on macrovascular and microvascular outcomes in patients with type 2 diabetes 
mellitus (the ADVANCE trial): a randomised controlled trial. Lancet. 2007;370(9590):829-40.

26. Patel A, MacMahon S, Chalmers J, Neal B, Billot L, Woodward M, et al. Intensive blood glucose control and vascular outcomes in patients with type 2 diabetes. N Engl J Med. 2008;358(24):2560-72.

27. Tesfaye S, Boulton AJ, Dyck PJ, Freeman R, Horowitz M, Kempler P, et al. Diabetic neuropathies: update on definitions, diagnostic criteria, estimation of severity, and treatments. Diabetes Care. 2010;33(10):2285-93.

28. Stefansdottir G, Zoungas S, Chalmers J, Kengne AP, Knol MJ, Leufkens HG, et al. Intensive glucose control and risk of cancer in patients with type 2 diabetes. Diabetologia. 2011;54(7):1608-14.

29. Fine JP, Gray RJ. A proportional hazards model for the subdistribution of a competing risk. J Am Stat Assoc. 1999;94:496-509.

30. Onega T, Baron JA, Johnsen SP, Pedersen L, Farkas DK, Sorensen HT. Cancer risk and subsequent survival after hospitalization for intermittent claudication. Cancer Epidemiol Biomarkers Prev. 2015;24(4):744-8.

31. Akai A, Shigematsu H, Miyata T, Maeda H, Onohara T, Sato O, et al. Increased incidence of cancer in japanese patients with critical limb ischemia. Ann Vasc Dis. 2019;12(2):182-6.

32. Sundboll J, Veres K, Horvath-Puho E, Adelborg K, Sorensen HT. Risk and Prognosis of Cancer After Lower Limb Arterial Thrombosis. Circulation. 2018;138(7):669-77.

33. Torre LA, Bray F, Siegel RL, Ferlay J, Lortet-Tieulent J, Jemal A. Global cancer statistics, 2012. CA Cancer J Clin. 2015;65(2):87-108.

34. Mohammedi K, Woodward M, Hirakawa Y, Zoungas S, Williams B, Lisheng $\mathrm{L}$, et al. Microvascular and macrovascular disease and risk for major peripheral arterial disease in patients with type 2 diabetes. Diabetes Care. 2016;39(10):1796-803.

35. Andersen ST, Witte DR, Dalsgaard EM, Andersen H, Nawroth P, Fleming T, et al. Risk factors for incident diabetic polyneuropathy in a cohort with screen-detected type 2 diabetes followed for 13 Years: ADDITIONDenmark. Diabetes Care. 2018;41(5):1068-75.

36. Barrera G. Oxidative stress and lipid peroxidation products in cancer progression and therapy. ISRN Oncol. 2012;2012:137289.

37. Kundu JK, Surh YJ. Emerging avenues linking inflammation and cancer. Free Radic Biol Med. 2012;52(9):2013-37.
38. Long M, de la Rojo Vega M, Wen Q, Bharara M, Jiang T, Zhang R, et al. An essential role of NRF2 in diabetic wound healing. Diabetes. 2016:65(3):780-93.

39. Nativel M, Schneider F, Saulnier PJ, Gand E, Ragot S, Meilhac O, et al. Prognostic Values of Inflammatory and Redox Status Biomarkers on the Risk of Major Lower-Extremity Artery Disease in Individuals With Type 2 Diabetes. Diabetes Care. 2018. https://doi.org/10.2337/dc18-0695.

40. Mohammedi K, Bellili-Munoz N, Marklund SL, Driss F, Le Nagard H, Patente TA, et al. Plasma extracellular superoxide dismutase concentration, allelic variations in the SOD3 gene and risk of myocardial infarction and all-cause mortality in people with type 1 and type 2 diabetes. Cardiovasc Diabetol. 2015;14:845.

41. Toya T, Sara JD, Corban MT, Taher R, Godo S, Herrmann J, et al. Assessment of peripheral endothelial function predicts future risk of solid-tumor cancer. Eur J Prev Cardiol. 2020;27(6):608-18.

42. Eelen G, de Zeeuw P, Treps L, Harjes U, Wong BW, Carmeliet P. Endothelial Cell Metabolism. Physiol Rev. 2018;98(1):3-58.

43. Lloyd-Jones DM, Hong Y, Labarthe D, Mozaffarian D, Appel LJ, Van Horn $L$, et al. Defining and setting national goals for cardiovascular health promotion and disease reduction: the American Heart Association's strategic Impact Goal through 2020 and beyond. Circulation. 2010;121(4):586-613.

44. Rasmussen-Torvik LJ, Shay CM, Abramson JG, Friedrich CA, Nettleton JA, Prizment $\mathrm{AE}$, et al. Ideal cardiovascular health is inversely associated with incident cancer: the Atherosclerosis Risk In Communities study. Circulation. 2013;127(12):1270-5.

45. Stubblefield MD, Burstein HJ, Burton AW, Custodio CM, Deng GE, Ho M, et al. NCCN task force report: management of neuropathy in cancer. J Natl Compr Canc Netw. 2009;7(Suppl 5):S1-26.

\section{Publisher's Note}

Springer Nature remains neutral with regard to jurisdictional claims in published maps and institutional affiliations.
Ready to submit your research? Choose BMC and benefit from:

- fast, convenient online submission

- thorough peer review by experienced researchers in your field

- rapid publication on acceptance

- support for research data, including large and complex data types

- gold Open Access which fosters wider collaboration and increased citations

- maximum visibility for your research: over $100 \mathrm{M}$ website views per year

At BMC, research is always in progress.

Learn more biomedcentral.com/submissions 